\title{
Mythos «Höhenkur» und die Gründung der Lungenliga
}

C. Schürer

Die Schweizerische Lungenliga besteht seit 100 Jahren. Anlässlich dieses Jubiläums soll ein kritischer Blick auf die Behandlungsgeschichte der Lungentuberkulose geworfen werden. sion war Karl Turban (1856-1935), womit am Anfang der Lungenliga ein schillernder Arzt steht. Turban, der «Tuberkulose-Tyrann» von Davos (Wilhelm Löffler), prägte die Sanatoriumstherapie in der Schweiz entscheidend. 1889 eröffnete er in Davos ein Privatsanatorium für eine wohlhabende internationale Klientel. Aus Deutschland brachte er das Konzept der geschlossenen Heilanstalt in die Schweiz, welches Peter Dettweiler (1837-1904) entwickelt hatte [3]. Gemäss Dettweiler musste der Arzt kraft seiner unangefochtenen Autorität die ganze Lebensführung der Patienten beherrschen, es war entscheidend, dass «das Sprechzimmer zum Beichtstuhle» wurde [4]. schweizerische Zentralkommission zur Bekämpfung der Tuberkulose» [1]. Die Zentralkommission ist die Vorläuferin der heutigen Lungenliga Schweiz. Die 100jährige Geschichte der Lungenliga beginnt somit mit dem Vorhaben, die verschiedenen kantonalen Massnahmen gegen die Tuberkulose besser zu koordinieren. Mehr als jeder zehnte Todesfall war damals auf Lungentuberkulose zurückzuführen [2]. Bei der Gründung der Zentralkommission stand die Bekämpfung ganz im Zeichen der Sanatoriumsbehandlung, welche in der Schweiz mit Vorliebe im Höhenklima durchgeführt wurde. Erster Vorsitzender der Zentralkommis-

\section{Die Theorie des heilsamen Höhenklimas}

Dettweilers rigides ärztliches Konzept wurde für die Heilstättenbewegung in Deutschland und der Schweiz grundlegend. Die Bewegung hatte zum Ziel, auch unbemittelten Tuberkulosekranken den Aufenthalt in einer Heilanstalt zu ermöglichen. Richtig ins Laufen gebracht wurde sie durch Robert Kochs Fehlschlag mit dem vermeintlichen Heilmittel Tuberkulin im Jahr 1890 [5]. Auch in der Schweiz wurde die Idee der
Korrespondenz: Christian Schürer Forschungsstelle für Sozialund Wirtschaftsgeschichte Universität Zürich

Rämistrasse 64 CH-8001 Zürich

E-Mail: cschuerer@freesurf.ch

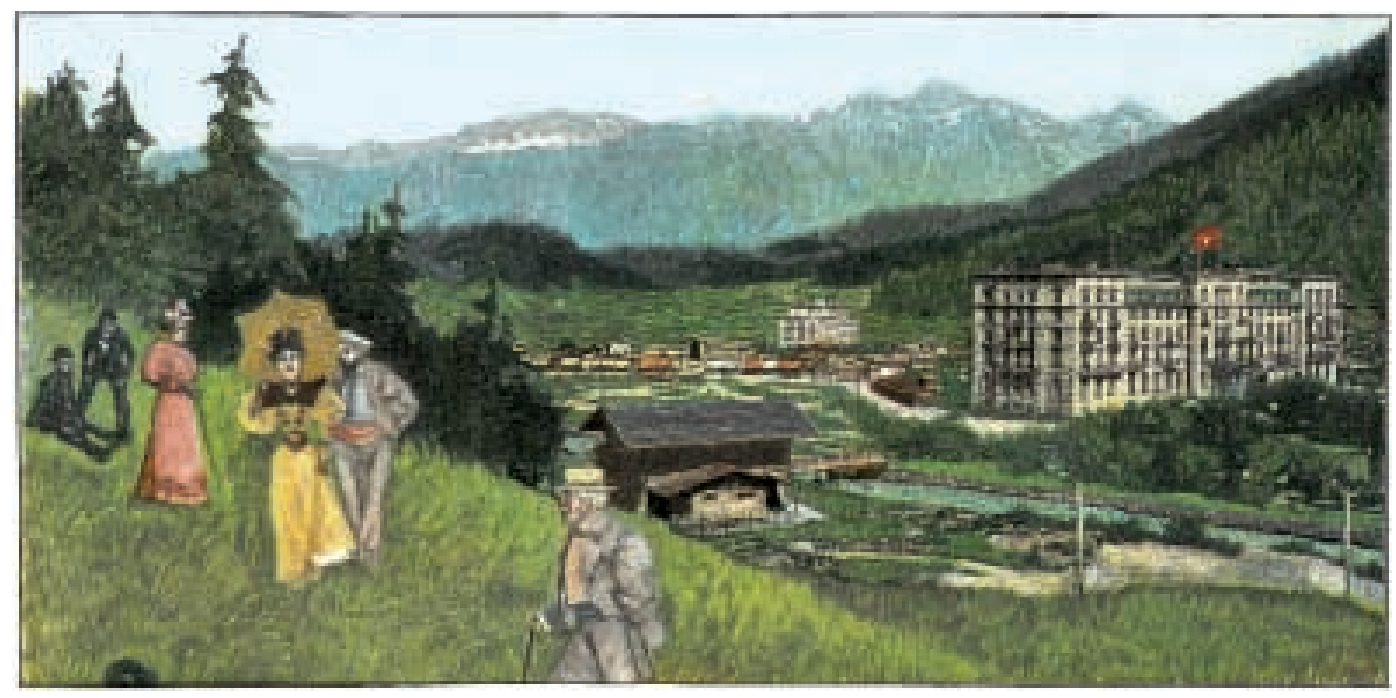

Kurgäste in Davos um 1900. Medizinhistorisches Institut der Universität Zürich: Archiv BS b. 
Volkssanatorien in den 1890er Jahren aufgegriffen. Erste Heilstätten entstanden [6]. Nicht nur humanitäre, sondern auch volkswirtschaftliche Überlegungen waren für den Bau von Volkssanatorien entscheidend. Jakob Laurenz Sonderegger (1825-1896), einer der einflussreichsten Schweizer Ärzte seiner Zeit, wies 1894 darauf hin, dass die Schwindsüchtigen «nicht rasch wegsterben wie bei der Cholera, sondern Jahre lang fortleben», und damit der bürgerlichen Gesellschaft zur Last fallen würden [7]. Die Heilstättenbewegung setzte sich deshalb zum Ziel, die Kranken wieder arbeitsfähig zu machen.

Wenn möglich wurden die Volkssanatorien im Höhenklima gebaut. So eröffnete die Basler Heilstätte ihre Tore in Davos, diejenige von Genf in Montana. Dies macht deutlich, wie verbreitet die Theorie des Davoser Arztes Alexander Spengler (1827-1901) war, dass das Höhenklima die Lungentuberkulose heilen könne. Spengler hatte der Theorie in den 1860er Jahren zum Durchbruch verholfen [8]. Ein wichtiger Pfeiler derselben kam indes schon 1875 durch eine Untersuchung des Winterthurer Bezirksarztes Emil Müller zum Einsturz. Entgegen der These Spenglers wies Müller nach, dass auch die im Höhenklima lebenden Menschen an Tuberkulose starben, dieses also keineswegs «immun» machte. Nichtsdestotrotz wurde das Höhenklima in der medizinischen Diskussion noch lange als heilkräftig erachtet. Immer mehr hochgelegene Orte ernannten sich selbst zu Höhenkurorten. Kur- und Sanatoriumsärzte - Vincent Barras spricht vom Typus des «entrepreneur médical» [9] - förderten mit ihrer Publikationstätigkeit diese Entwicklung entscheidend.

\section{Die Patienten als «Apostel der Hygiene»}

Die aus ärmeren Schichten stammenden Patienten der Volksheilstätten sollten zu «Studenten der Gesundheit» (Sonderegger) werden, denen nicht nur bakteriologische Hygieneregeln wie Spuckdisziplin, sondern auch bürgerliche Moralvorstellungen eingeimpft wurden. Die Volkssanatorien standen indes schon zur Zeit der Gründung der Zentralkommission in der Kritik. Dr. E. Fischer fragte 1902 im «CorrespondenzBlatt für Schweizer Aerzte»: "Thun wir unsere Pflicht im Kampfe gegen die Schwindsucht als Volkskrankheit?» [10]. Ein Vergleich von Statistiken über die Behandlungserfolge von Heilanstalten fällt für ihn ernüchternd aus, umso mehr, als die Volkssanatorien «nur Kranke im Initialstadium der Phthise» aufnahmen. Schwer- kranke blieben aussen vor. Kein Mensch kümmere sich um sie, beklagt Fischer. Die Volksheilstätten versuchten die ab 1900 stärker wirkende Kritik zu parieren, indem sie immer weniger die Heilung der Kranken, sondern die hygienische Volkserziehung in den Vordergrund rückten. Die Entlassenen sollten als «Apostel der Hygiene» in ihre Kreise zurückkehren, die sozialen Verhältnisse der ärmeren Bevölkerungsschichten wurden hingegen kaum thematisiert [11]. Verschiedene Studien weisen darauf hin, dass es nicht die Lungenkur, sondern die Verbesserung der Lebens- und Arbeitsbedingungen war, welche den Rückgang der Tuberkulosesterblichkeit zur Folge hatte. Die Medizin im engeren Sinne reduzierte die Tuberkulose nachweislich erst ab 1948 mit der Chemotherapie [12].

\section{Literatur}

1 Ganguillet F. Organisation der Tuberkulosebekämpfung in der Schweiz. In: Schweizerische Zentralkommission zur Bekämpfung der Tuberkulose (Hrsg.). Die Tuberkulose und ihre Bekämpfung in der Schweiz. Bern; 1917.

2 Ritzmann I. Hausordnung und Liegekur. Vom Volkssanatorium zur Spezialklinik: 100 Jahre Zürcher Höhenklinik Wald. Zürich: Chronos; 1998.

3 Turban K. Lebenskampf. Die Selbstbiographie eines Arztes. Leipzig; 1935.

4 Dettweiler P. Die Behandlung der Lungenschwindsucht in geschlossenen Heilanstalten mit besonderer Beziehung auf Falkenstein i/T. Zweite veränderte Auflage. Berlin; 1884.

5 Condrau F. Lungenheilanstalt und Patientenschicksal. Sozialgeschichte der Tuberkulose in Deutschland und England im späten 19. und frühen 20. Jahrhundert. Göttingen: Vandenhoeck \& Ruprecht; 2000.

6 Rüttimann B. Volkssanatorien in der Schweiz. Schweiz Ärztezeitung 2000;81:2821-4.

7 Sonderegger JL. Tuberkulose und Heilstätten für Brustkranke in der Schweiz. St. Gallen; 1894.

8 Schenk R. Geschichte des heilklimatischen Kurortes Davos im Spiegel seiner Tagespublizistik. Bochum: Universitätsverlag Dr. N. Brockmeyer; 1991.

9 Barras V. Histoire d'une station climatérique, Montana, Canton du Valais. Rev Méd Suisse Romande 1994;114:361-71.

10 Fischer E. Thun wir unsere Pflicht im Kampfe gegen die Schwindsucht als Volkskrankheit? Correspondenz-Blatt für Schweizer Aerzte 1902;32:681-91.

11 Gredig D. Tuberkulosefürsorge in der Schweiz. Zur Professionsgeschichte der Sozialen Arbeit. Die Tuberkulosefürsorgestelle Basel 1906-1961. Bern/Stuttgart/Wien: Paul Haupt; 2000.

12 McKeown T. The Role of Medicine. Dream, Mirage oder Nemesis? Oxford: Basil Blackwell; 1979. 